\title{
Nominal Logic with Equations Only
}

\author{
Ranald Clouston * \\ Logic and Computation Group, Research School of Computer Science, Canberra, ACT 0200, Australia \\ ranald.clouston@anu.edu.au
}

\begin{abstract}
Many formal systems, particularly in computer science, may be captured by equations modulated by side conditions asserting the "freshness of names"; these can be reasoned about with Nominal Equational Logic (NEL). Like most logics of this sort NEL employs this notion of freshness as a first class logical connective. However, this can become inconvenient when attempting to translate results from standard equational logic to the nominal setting. This paper presents proof rules for a logic whose only connectives are equations, which we call Nominal Equation-only Logic (NEoL). We prove that NEoL is just as expressive as NEL. We then give a simple description of equality in the empty NEoL-theory, then extend that result to describe freshness in the empty NEL-theory.
\end{abstract}

\section{Introduction}

Many formal systems, particularly in computer science, may be captured via equations modulated by side conditions asserting certain names are fresh for (not in the free names of) certain metavariables:

First-order logic: $\Phi \supset(\forall a . \Psi)=\forall a .(\Phi \supset \Psi)$ if $a$ is fresh for $\Phi$;

$\lambda$-calculus: $\lambda a \cdot f a=\eta f$ if $a$ is fresh for $f$;

$\pi$-calculus: $(v a x) \mid y=v a(x \mid y)$ if $a$ is fresh for $y$.

We may express such modulated equations, and hence reason formally about the systems described by them, with Nominal Equational Logic (NEL) [5]. NEL-theories can also express the notions of binding and $\alpha$-equivalence such systems exhibit [3]. NEL generalises standard equational logic by employing the nominal sets model [11], a refinement of the earlier Fraenkel-Mostowski sets model [9], where the manipulation of names is modelled by the action of permutations.

In the examples above the 'fresh for' relation, represented in NEL by the symbol ' $\not$ ', is attached to metavariables as a side condition to the equations. However this relation generalises naturally and conveniently to a relation asserting certain names are fresh for certain terms. As such, in NEL and other nominal logics, $\not Æ$ is treated as a first class logical connective, rather than merely being used in side conditions.

Standard equational logic is an extremely well studied system (e.g. [1, Cha. 3]), and NEL's development philosophy was to maintain as close a relationship as possible to this standard account, so that well known results can be transferred to a setting with names and binding with a minimum of difficulty. There are two orthogonal ways in which this can be done. The first is to translate techniques and results from equational logic to NEL, such as term rewriting, Lawvere theories, algebras for a monad, and Birkhoffstyle closure results. The second is to combine NEL with other extensions of equational logic, of which a multitude exist - partial, order-sorted, conditional, membership, fuzzy, and so forth.

\footnotetext{
${ }^{*}$ The author gratefully acknowledges the advice of Andrew Pitts and the reviewers, and the support of the Woolf Fisher Trust.
}

H. Geuvers, G. Nadathur (Eds): LFMTP 2011

EPTCS 71, 2011, pp. 44-57 doi 10.4204/EPTCS.71.4

(c) R. Clouston 
There is a tension here, as we wish to exploit known results of equational logic while also having the convenience of $\not \nVdash$ as a first class logical connective, so that NEL is no longer really an equational logic at all. Consider the case of Lawvere theories [10]. In this category theoretic view of equational logic, an equational theory is mapped to a classifying category, whose arrows are tuples of terms, and the equality of arrows is asserted to correspond to the provable equality of terms in the theory. This is intuitive because equality has a clear meaning in the category theoretic setting, as it must in any mathematical setting. This is not true of freshness, which is a notion bespoke to the nominal sets model with no obvious meaning in, say, standard category theory.

Fortunately, it is known that in a variety of contexts freshness judgements can be translated into equally expressive equations with freshness side conditions. The earliest such result to our knowledge is for the related logic of Nominal Algebra (see e.g. [8, Lem. 4.5.1]); the corresponding result for NEL is Lem. 3.8 of this paper. We can therefore treat $\not \Varangle$ as syntactic sugar, and justifiably call NEL an equational logic.

However, working with the standard proof rules for NEL, in which $\not$ is extensively used as a first class connective, may still be highly inconvenient when trying to exploit known results of equational logic. Developing an analogue of Lawvere theories for NEL in [4] required some complex proofs relating logical derivations in NEL to category theoretic properties. Having freshness judgements in those proof derivations with no obvious category theoretic interpretation, and therefore being forced to apply the conversion to equations of Lem. 3.8 each time, would have been extremely laborious, and render the proofs obscure. Instead, the development of Nominal Lawvere theories used alternative proof rules for NEL that employ equations only, relegating freshness assertions back to the side conditions.

In this paper we present these proof rules (in slightly modified form), which we call Nominal Equation-only Logic (NEoL), and in Sec. 4 we show that NEoL and NEL coincide. This result, which until now has only appeared in Sec. 5.5 of the author's thesis [2], is crucial to the published proof that Nominal Lawvere theories correspond to NEL-theories. We posit that NEoL will continue to be convenient when applying standard equational logic results to names and binding, even as NEL remains the more convenient system for applications.

In standard equational logic two terms are provably equal to each other in the empty theory if and only if they are syntactically identical. Sec. [5 presents for the first time a simple syntax-directed description of equality in the empty theory for NEoL. Cor. 5.5 extends this result to NEL to give a description of freshness in the empty theory. Finally Sec. 6 compares NEoL and NEL to three related notions in the literature of equational logic over nominal sets.

\section{Nominal Sets}

We will first introduce the basic mathematics of the nominal sets model, which will be necessary for the presentation of the syntax of Nominal Equational Logic in the next section.

Fix a countably infinite set $\mathbb{A}$ of atoms, which we will use as names. The set Perm of (finite) permutations consists of all bijections $\pi: \mathbb{A} \rightarrow \mathbb{A}$ whose domain

$$
\operatorname{supp}(\pi) \triangleq\{a \mid \pi(a) \neq a\}
$$

is finite. Perm is a group with multiplication as permutation composition $\pi^{\prime} \pi(a)=\pi^{\prime}(\pi(a))$, and identity as the permutation $\imath$ leaving all atoms unchanged. Perm is generated by transpositions $(a b)$ that map $a$ to $b, b$ to $a$ and leave all other atoms unchanged. We will make particular use of permutations known as 
generalised transpositions [5, Lem. 10.2]. Let

$$
\mathbb{A}^{(n)} \triangleq\left\{\left(a_{1}, \ldots, a_{n}\right) \in \mathbb{A}^{n} \mid a_{i} \neq a_{j} \text { for } 1 \leq i<j \leq n\right\} .
$$

All the tuples of atoms we use in this paper will be from this set. Take $\vec{a}=\left(a_{1}, \ldots, a_{n}\right), \vec{a}^{\prime}=\left(a_{1}^{\prime}, \ldots, a_{n}^{\prime}\right) \in$ $\mathbb{A}^{(n)}$ with disjoint underlying sets. Then we define their generalised transposition as

$$
\left(\vec{a} \vec{a}^{\prime}\right) \triangleq\left(a_{1} a_{1}^{\prime}\right) \cdots\left(a_{n} a_{n}^{\prime}\right)
$$

A Perm-set is a set $X$ equipped with a function, or Perm-action, $(\pi, x) \mapsto \pi \cdot x$ from Perm $\times X$ to $X$ such that $\imath \cdot x=x$ and $\pi \cdot\left(\pi^{\prime} \cdot x\right)=\pi \pi^{\prime} \cdot x$.

Given such a Perm-set $X$ we say that a set of atoms $\bar{a} \subseteq \mathbb{A}$ supports $x \in X$ if for all $\pi \in$ Perm, $\operatorname{supp}(\pi) \cap \bar{a}=\emptyset$ implies that $\pi \cdot x=x$.

Definition 2.1. A nominal set is a Perm-set $X$ with the finite support property: for each $x \in X$ there exists some finite $\bar{a} \subseteq \mathbb{A}$ supporting $x$.

If an element $x$ is finitely supported then there is a unique least such support set [9, Prop. 3.4], which we write $\operatorname{supp}(x)$ and call the support of $x$. This may be read as the set of free names of a term. If $\bar{a} \cap \operatorname{supp}(x)=\emptyset$ for some $\bar{a} \subseteq \mathbb{A}$ we say that $\bar{a}$ is fresh for $x$ and write $\bar{a} \# x$, capturing the not free in relation.

Example 2.2. (i) Any set becomes a nominal set under the trivial Perm-action $\pi \cdot x=x$, with finite support property $\operatorname{supp}(x)=\emptyset$;

(ii) $\mathbb{A}$ is a nominal set with Perm-action $\pi \cdot a=\pi(a)$ and $\operatorname{supp}(a)=\{a\}$;

(iii) Perm is a nominal set with Perm-action $\pi \cdot \pi^{\prime}=\pi \pi^{\prime} \pi^{-1}$ and support as in (1);

(iv) Finite products of nominal sets are themselves nominal sets given the element-wise Perm-action and $\operatorname{supp}\left(x_{1}, \ldots, x_{n}\right)=\bigcup_{1 \leq i \leq n} \operatorname{supp}\left(x_{i}\right)$;

(v) $\mathbb{A}^{(n)}$, and the set of finite sets of atoms $\mathscr{P}_{\text {fin }}(\mathbb{A})$, are nominal sets given the element-wise Permactions. Supports correspond to underlying sets.

Lemma 2.3. Given a nominal set $X$, element $x \in X$, and permutations $\pi, \pi^{\prime} \in$ Perm,

(i) Given finite $\bar{a} \subseteq \mathbb{A}, \bar{a} \# x$ implies $\pi \cdot \bar{a} \# \pi \cdot x$;

(ii) The disagreement set of $\pi$ and $\pi^{\prime}$ is

$$
d s\left(\pi, \pi^{\prime}\right) \triangleq\left\{a \mid \pi(a) \neq \pi^{\prime}(a)\right\} .
$$

Then $d s\left(\pi, \pi^{\prime}\right) \#$ implies $\pi \cdot x=\pi^{\prime} \cdot x$.

Proof. [12, Lem. 3.7] and [5, Lem. 7.3(iv)].

Given Perm-sets $X, Y$ we can define a Perm-action on functions $f: X \rightarrow Y$ by

$$
(\pi \cdot f)(x) \triangleq \pi \cdot\left(f\left(\pi^{-1} \cdot x\right)\right)
$$

Hence if $f$ maps $x \mapsto y$ then $\pi \cdot f$ maps $\pi \cdot x \mapsto \pi \cdot y$. A finitely supported function is a function with finite support under this definition; this terminology is necessary as even where $X, Y$ are nominal sets not all functions between them have this property. In the particular case that $f$ has empty support, we call it equivariant. This is equivalent to the condition that $\pi \cdot(f(x))=f(\pi \cdot x)$ for all permutations $\pi$. 


\section{Nominal Equational Logic}

This section presents syntax and proof rules for Nominal Equational Logic (NEL) [5]. In fact it is sometimes useful to mildly generalise NEL so that its sorts form a nominal set, rather than a set, of sorts, as is done in [4]. However this does not materially effect the results of this paper and so the simpler original presentation is here used.

Definition 3.1. A NEL-signature $\Sigma$ is specified by

(i) a set Sort $\Sigma$, whose elements are called the sorts of $\Sigma$;

(ii) a nominal set $O \mathrm{p}_{\Sigma}$, whose elements are called the operation symbols of $\Sigma$;

(iii) an equivariant typing function mapping each operation symbol $o p \in O \mathrm{p}_{\Sigma}$ to a type consisting of a finite list $\overrightarrow{\mathrm{s}}=\left(\mathrm{s}_{1} \ldots, \mathrm{s}_{n}\right)$ of sorts of $\Sigma$ and another $\mathrm{s} \in \operatorname{Sort}_{\Sigma}$. We write this op $: \overrightarrow{\mathrm{s}} \rightarrow \mathrm{s}$. Where $n=0$ we write $o p: \mathrm{s}$. Equivariance of the typing function means that each $o p, \pi \cdot o p$ have the same type.

Example 3.2. A NEL-signature for the untyped $\lambda$-calculus can be defined by letting our sorts be the singleton $\{\mathrm{tm}\}$ and operation symbols be

$$
\left\{\operatorname{var}_{a} \mid a \in \mathbb{A}\right\} \cup\left\{\operatorname{lam}_{a} \mid a \in \mathbb{A}\right\} \cup\{a p p\}
$$

representing object-level variables, lambda-abstractions and application respectively. The Perm-action on these operations symbols is

$$
\pi \cdot \operatorname{var}_{a} \triangleq \operatorname{var}_{\pi(a)}, \quad \pi \cdot \operatorname{lam}_{a} \triangleq \operatorname{lam}_{\pi(a)}, \quad \pi \cdot a p p \triangleq a p p
$$

The typing function is

$$
\operatorname{var}_{a}: \mathrm{tm}, \quad \operatorname{lam}_{a}:(\mathrm{tm}) \rightarrow \mathrm{tm}, \quad \text { app }:(\mathrm{tm}, \mathrm{tm}) \rightarrow \mathrm{tm} .
$$

Definition 3.3. Fix a countably infinite set Var of variables. Then the terms over $\Sigma$ are

$$
t::=\pi x \mid \text { opt } \cdots t
$$

for $\pi \in$ Perm, $x \in \operatorname{Var}$ and $o p \in O p_{\Sigma}$. We call $\pi x$ a suspension and write $\imath x$ simply as $x$. We call opt $t_{1} \cdots t_{n}$ a constructed term.

The sorting environments $\mathrm{SE}_{\Sigma}$ are partial functions $\Gamma: \operatorname{Var} \rightarrow$ Sort $_{\Sigma}$ with finite domain. We define the set $\Sigma_{\mathrm{s}}(\Gamma)$ of terms of sort $\mathrm{s}$ in $\Gamma$ by

(i) if $\pi \in$ Perm and $x \in \operatorname{dom}(\Gamma)$ then $\pi x \in \Sigma_{\Gamma(x)}(\Gamma)$;

(ii) if $o p:\left(\mathrm{s}_{1}, \ldots, \mathrm{s}_{n}\right) \rightarrow \mathrm{s}$ and $t_{i} \in \Sigma_{\mathrm{s}_{i}}(\Gamma)$ for $1 \leq i \leq n$, then $o p t_{1} \cdots t_{n} \in \Sigma_{\mathrm{s}}(\Gamma)$.

The object-level Perm-action on terms, $\left(\pi, t \in \Sigma_{\mathrm{s}}(\Gamma)\right) \mapsto \pi * t \in \Sigma_{\mathrm{s}}(\Gamma)$, is

$$
\begin{aligned}
\pi *\left(\pi^{\prime} x\right) & \triangleq \pi \pi^{\prime} x ; \\
\pi *\left(o p t_{1} \cdots t_{n}\right) & \triangleq(\pi \cdot o p)\left(\pi * t_{1}\right) \cdots\left(\pi * t_{n}\right) .
\end{aligned}
$$

This action is used in the definition of substitution: given $\Gamma, \Gamma^{\prime} \in \mathrm{SE}_{\Sigma}$, a substitution $\sigma: \Gamma \rightarrow \Gamma^{\prime}$ is a map from each $x \in \operatorname{dom}(\Gamma)$ to $\sigma(x) \in \Sigma_{\Gamma(x)}\left(\Gamma^{\prime}\right)$. Given a term $t \in \Sigma_{\mathbf{s}}(\Gamma)$, the term $t\{\sigma\} \in \Sigma_{\mathbf{s}}\left(\Gamma^{\prime}\right)$ is defined by

$$
\begin{aligned}
(\pi x)\{\sigma\} & \triangleq \pi * \sigma(x) ; \\
\left(o p t_{1} \cdots t_{n}\right)\{\sigma\} & \triangleq \operatorname{opt}_{1}\{\sigma\} \cdots t_{n}\{\sigma\} .
\end{aligned}
$$


We will write the single term substitution that replaces the variable $x$ with the term $t$ and leaves all other variables unchanged as $(t / x)$.

Terms are not in general finitely supported under the Perm-action (2). However there is another notion of Perm-action on terms which has this property, so that each $\Sigma_{\mathrm{s}}(\Gamma)$ is a nominal set. The metalevel Perm-action on terms, $\left(\pi, t \in \Sigma_{\mathrm{s}}(\Gamma)\right) \mapsto \pi \cdot t \in \Sigma_{\mathrm{s}}(\Gamma)$, is

$$
\begin{aligned}
\pi \cdot\left(\pi^{\prime} x\right) & \triangleq \pi \pi^{\prime} \pi^{-1} x ; \\
\pi \cdot\left(\text { opt }_{1} \cdots t_{n}\right) & \triangleq(\pi \cdot o p)\left(\pi \cdot t_{1}\right) \cdots\left(\pi \cdot t_{n}\right) .
\end{aligned}
$$

The following Lemma relates these notions:

Lemma 3.4. Given $t \in \Sigma_{\mathrm{s}}(\Gamma)$, $\pi \in$ Perm and a substitution $\sigma$,

(i) $\pi *(t\{\sigma\})=(\pi * t)\{\sigma\}$;

(ii) $\pi \cdot t=\pi * t\left\{\pi^{-1}-\right\}$, where $\left(\pi^{-1}-\right)$ is the substitution mapping each $x \mapsto \pi^{-1} x$.

Proof. Easy inductions on the structure of $t$; see [5, Lem 5.2 \& (30)] or [7, Lem. 2.3].

The freshness environments $\mathrm{FE}_{\Sigma}$ are partial functions $\nabla$ with finite domain on Var, mapping each $x \in \operatorname{dom}(\nabla)$ to a pair $(\bar{a}, \mathrm{~s})$ where $\bar{a} \in \mathscr{P}_{\text {fin }}(\mathbb{A})$ and $\mathrm{s} \in \operatorname{Sort}_{\Sigma} . \mathrm{FE}_{\Sigma}$ is then a nominal set under the action $(\pi \cdot \nabla)(x)=(\pi \cdot \bar{a}, \mathrm{~s}) ; \operatorname{supp}(\nabla)$ is $\bigcup_{x \in \operatorname{dom}(\nabla)} \operatorname{supp}(\nabla(x))$. If $\nabla\left(x_{i}\right)=\left(\bar{a}_{i}, \mathrm{~s}_{i}\right)$ for $1 \leq i \leq n$ we write $\nabla$ as

$$
\left(\bar{a}_{1} \not Æ x_{1}: \mathrm{s}_{1}, \ldots, \bar{a}_{n} \not Æ x_{n}: \mathrm{s}_{n}\right) \text {. }
$$

The intended meaning is that $\bar{a}_{i}$ is fresh for $x_{i}$, which has sort $\mathrm{s}_{i}$. These will capture the freshness side conditions we discussed in the introduction. Each $\nabla \in \mathrm{FE}_{\Sigma}$ gives rise to a sorting environment $\nabla: \in \mathrm{SE}_{\Sigma}$ by taking the second projection. We will abbreviate $\{a\} \not Æ x: \mathrm{s}$ as $a \not Æ x: \mathrm{s}$ and $\emptyset \not 火 x: \mathrm{s}$ as $x$ : s.

Definition 3.5. A NEL-judgement has the form

$$
\nabla \vdash \bar{a} \not \sharp t \approx t^{\prime}: \mathrm{s}
$$

where $\nabla \in \mathrm{FE}_{\Sigma}, \bar{a} \in \mathscr{P}_{\text {fin }}(\mathbb{A}), \mathrm{s} \in \operatorname{Sort}_{\Sigma}$ and $t, t^{\prime} \in \Sigma_{\mathrm{s}}\left(\nabla^{:}\right)$. We will abbreviate $\nabla \vdash \bar{a} \not Æ t \approx t: \mathrm{s}$ as $\nabla \vdash \bar{a} \not \sharp t: \mathrm{s}$ and $\nabla \vdash \emptyset \not t t \approx t^{\prime}: \mathrm{s}$ as $\nabla \vdash t \approx t^{\prime}: \mathrm{s}$.

A $N E L$-theory $\mathbb{T}$ is a collection of such judgements, which we call its axioms.

Example 3.6. The axioms for $\alpha \beta \eta$-equivalence over the untyped $\lambda$-calculus (Ex. 3.2), adapting [8] Ex. 2.15], are

( $\alpha)(x: \mathrm{tm}) \vdash a \not t \operatorname{lam}_{a} x: \mathrm{tm}$

$\left(\beta_{1}\right)(a \not t x: \mathrm{tm}, y: \mathrm{tm}) \vdash \operatorname{app}\left(\operatorname{lam}_{a} x\right) y \approx x: \mathrm{tm}$

$\left(\beta_{2}\right)(y: \mathrm{tm}) \vdash \operatorname{app}\left(\operatorname{lam}_{a} v_{a r}\right) y \approx y: \mathrm{tm}$

$\left(\beta_{3}\right)(x: \mathrm{tm}, b \not t y: \mathrm{tm}) \vdash \operatorname{app}\left(\operatorname{lam}_{a}\left(\operatorname{lam}_{b} x\right)\right) y \approx \operatorname{lam}_{b}\left(\operatorname{app}\left(\operatorname{lam}_{a} x\right) y\right): \mathrm{tm}$

$\left(\beta_{4}\right)\left(x_{1}: \mathrm{tm}, x_{2}: \mathrm{tm}, y: \mathrm{tm}\right) \vdash \operatorname{app}\left(\operatorname{lam}_{a}\left(\operatorname{app}_{1} x_{2}\right)\right) y \approx \operatorname{app}\left(\operatorname{app}\left(\operatorname{lam}_{a} x_{1}\right) y\right)\left(\operatorname{app}\left(\operatorname{lam}_{a} x_{2}\right) y\right): \mathrm{tm}$

$\left(\beta_{5}\right)(b \not t x: \mathrm{tm}) \vdash \operatorname{app}\left(\operatorname{lam}_{a} x\right) \operatorname{var}_{b} \approx(a b) x: \mathrm{tm}$

( $\eta)(a \not 火 x: \mathrm{tm}) \vdash \operatorname{lam}_{a}\left(\right.$ appxvar $\left._{a}\right) \approx x: \mathrm{tm}$. 


$$
\begin{aligned}
& (\mathrm{REFL}) \frac{\nabla \vdash \bar{a} \not t t \approx t^{\prime}: \mathrm{s}}{\nabla \vdash t \approx t: \mathrm{s}} \nabla \in \mathrm{FE}_{\Sigma}, t \in \Sigma_{\mathrm{s}}(\nabla:) \quad(\mathrm{SYMM}) \frac{\nabla \vdash \bar{a} \not t t^{\prime} \approx t: \mathrm{s}}{\nabla} \\
& \text { (TRANS) } \frac{\nabla \vdash \bar{a}_{1} \not Æ t \approx t^{\prime}: \mathrm{s} \quad \nabla \vdash \bar{a}_{2} \not Æ t^{\prime} \approx t^{\prime \prime}: \mathrm{s}}{\nabla \vdash\left(\bar{a}_{1} \cup \bar{a}_{2}\right) \not Æ t \approx t^{\prime \prime}: \mathrm{s}} \quad \text { (WEAK) } \frac{\nabla \vdash \bar{a} \not Æ t \approx t^{\prime}: \mathrm{s}}{\nabla^{\prime} \vdash \bar{a} \not Æ t \approx t^{\prime}: \mathrm{s}} \nabla \leq \nabla^{\prime} \in \mathrm{FE}_{\Sigma} \\
& (\operatorname{SUBST}) \frac{\nabla^{\prime} \vdash \sigma \approx \sigma^{\prime}: \nabla \quad \nabla \vdash \bar{a} \not Æ t \approx t^{\prime}: \mathrm{s}}{\nabla^{\prime} \vdash \bar{a} \not Æ t\{\sigma\} \approx t^{\prime}\left\{\sigma^{\prime}\right\}: \mathrm{s}} \sigma, \sigma^{\prime}: \nabla: \rightarrow\left(\nabla^{\prime}\right):
\end{aligned}
$$

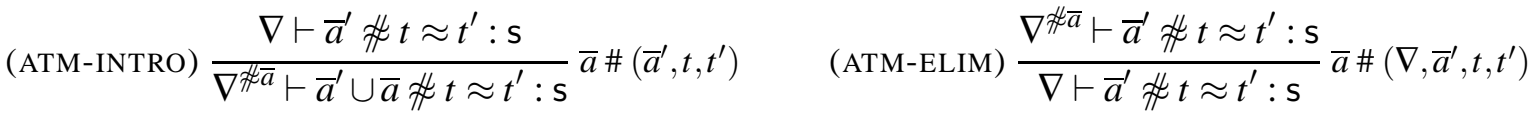

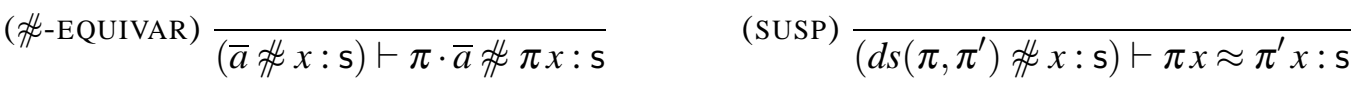

Figure 1: Proof rules for NEL

Definition 3.7. [Logical Consequence] The set of theorems of a NEL-theory $\mathbb{T}$ is the least set of judgements containing the axioms of $\mathbb{T}$ and closed under the rules of Fig. 1] We write

$$
\nabla \vdash_{\mathbb{T}} \bar{a} \not \nVdash t \approx t^{\prime}: \mathrm{s}
$$

to indicate that the judgement is a theorem of $\mathbb{T}$.

Fig. 1 uses the following new pieces of notation:

- In (WEAK) the relation $\nabla \leq \nabla^{\prime}$ holds if $\operatorname{dom}(\nabla) \subseteq \operatorname{dom}\left(\nabla^{\prime}\right)$ and for all $x \in \operatorname{dom}(\nabla)$ we have $\nabla(x)=$ $(\bar{a}, \mathrm{~s})$ and $\nabla^{\prime}(x)=\left(\bar{a}^{\prime}, \mathrm{s}\right)$ so that $\bar{a} \subseteq \bar{a}^{\prime}$.

- In rule (SUBST)

$$
\nabla^{\prime} \vdash \sigma \approx \sigma^{\prime}: \nabla
$$

stands for the hypotheses $\nabla^{\prime} \vdash \bar{a}_{i} \not Æ \sigma\left(x_{i}\right) \approx \sigma^{\prime}\left(x_{i}\right): \mathrm{s}_{i}$ for $1 \leq i \leq n$, where $\nabla$ is as (5).

- In (ATM-INTRO) and (ATM-ELIM), if $\bar{a}$ is a finite set of atoms and $\nabla$ is as (5) then

$$
\nabla \not Æ a \bar{a} \triangleq\left(\bar{a}_{1} \cup \bar{a} \not 火 x_{1}: \mathrm{s}_{1}, \ldots, \bar{a}_{n} \cup \bar{a} \not Æ x_{n}: \mathrm{s}_{n}\right) \text {. }
$$

Note also that (ATM-INTRO) and (ATM-ELIM) carry side conditions relating to freshness. These do not refer to the freshness connective $\not$ internal to the logic. Rather, they refer to the not-in-the-support-of relation \# of Def. 2.1 over the nominal sets $\mathrm{FE}_{\Sigma}, \mathscr{P}_{\text {fin }}(\mathbb{A})$, and $\Sigma_{\mathrm{s}}\left(\nabla^{*}\right)$ with respect to the action (4).

In [5] semantics are given for NEL, in which sorts are interpreted as nominal sets and operation symbols as finitely supported functions between them. The proof rules of Fig. 1 1 are shown to be sound and complete for that semantics. In this paper, however, we will work purely in terms of NEL's proof theory.

The next Lemma shows how freshness judgements may be translated into equivalent equational judgements in NEL. This will be crucial to the results of the next section, where we will get rid of freshness judgements entirely. 
Lemma 3.8. Given $t \in \Sigma_{\mathrm{s}}\left(\nabla^{:}\right)$and $\bar{a} \in \mathscr{P}_{\text {fin }}(\mathbb{A})$,

$$
\nabla \vdash_{\mathbb{T}} \bar{a} \not Æ t: \mathrm{s} \Leftrightarrow \nabla \not \sharp \operatorname{supp}\left(\vec{a}^{\prime}\right) \vdash_{\mathbb{T}} t \approx\left(\vec{a} \vec{a}^{\prime}\right) * t: \mathrm{s}
$$

where $\vec{a} \in \mathbb{A}^{(n)}$ is an ordering of $\bar{a}$ and $\vec{a}^{\prime} \in \mathbb{A}^{(n)}$ is a tuple of the same size such that supp $\left(\vec{a}^{\prime}\right) \#(\nabla, \bar{a}, t)$.

Proof. Left-to-right: $\nabla \not s u p p\left(\vec{a}^{\prime}\right) \vdash \bar{a} \cup \operatorname{supp}\left(\vec{a}^{\prime}\right) \not Æ t: \mathrm{s}$ by (ATM-INTRO); $\left(\bar{a} \cup \operatorname{supp}\left(\vec{a}^{\prime}\right) \not Æ x: \mathrm{s}\right) \vdash x \approx\left(\vec{a} \vec{a}^{\prime}\right) x$ : s by (SUSP); the result then follows by (SUBST) and (3).

Right-to-left: $\nabla \not$ supp $\left(\vec{a}^{\prime}\right) \vdash \operatorname{supp}\left(\vec{a}^{\prime}\right) \not Æ t: \mathrm{s}$ by (REFL) and (ATM-INTRO); $\left(\operatorname{supp}\left(\vec{a}^{\prime}\right) \not \sharp x: \mathrm{s}\right) \vdash \bar{a} \not Æ$

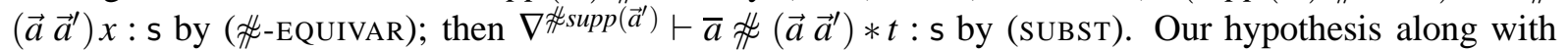
(TRANS) and (SYMM) gives us $\nabla \not$ supp $\left(\vec{a}^{\prime}\right) \vdash \bar{a} \not Æ t: \mathrm{s}$; the result then follows by (ATM-ELIM).

Example 3.9. The rule for $\alpha$-equivalence for the untyped $\lambda$-calculus in Ex. 3.6

$$
(x: \mathrm{tm}) \vdash a \not t \operatorname{lam}_{a} x: \mathrm{tm}
$$

is equivalent to

$$
(b \not t x: \mathrm{tm}) \vdash \operatorname{lam}_{a} x \approx \operatorname{lam}_{b}(a b) x: \mathrm{tm} .
$$

\section{Nominal Equation-only Logic}

This section presents syntax and proof rules for NEL without freshness connectives to the right of the turnstile $\vdash$. We call this Nominal Equation-only Logic (NEoL), and show that it is just as expressive as NEL.

Note that the previously published version of NEoL [4] included a rule called (PERM) that was somewhat unwieldy. This paper improves the presentation of NEoL by replacing (PERM) with a special case (SUSP), and then derives (PERM) as Lem. 4.6.

Definition 4.1. A NEoL-judgement has the form

$$
\nabla \vdash t \approx t^{\prime}: \mathrm{s}
$$

where $\nabla \in \mathrm{FE}_{\Sigma}, \mathrm{s} \in \operatorname{Sort}_{\Sigma}$ and $t, t^{\prime} \in \Sigma_{\mathbf{s}}\left(\nabla^{:}\right)$. Note that NEoL-judgements are also NEL-judgements (Def. 3.5).

A NEoL-theory $\mathbb{T}$ is a collection of such judgements, called its axioms.

Definition 4.2. The set of theorems of a NEoL-theory $\mathbb{T}$ is the least set of judgements containing the axioms of $\mathbb{T}$ and closed under the rules of Fig. 2, We write

$$
\nabla \vdash_{\mathbb{T}}^{o} t \approx t^{\prime}: \mathrm{s}
$$

to indicate that the judgement is a theorem of $\mathbb{T}$.

Say $\nabla$ is as (5). Then the rule $\left(\operatorname{SUBST}^{\circ}\right)$ in Fig. 2 uses the following new pieces of notation (ref. (7) and Lem. 3.8):

- $\nabla^{\prime} \vdash \sigma \approx \sigma^{\prime}$ stands for the hypotheses $\nabla^{\prime} \vdash \sigma\left(x_{i}\right) \approx \sigma^{\prime}\left(x_{i}\right): \mathrm{s}_{i}$ for $1 \leq i \leq n$;

- $\nabla^{\prime} \vdash \sigma: \nabla$ stands for the hypotheses

$$
\left(\nabla^{\prime}\right) \not \sharp \operatorname{supp}\left(\vec{a}_{i}^{\prime}\right) \vdash \sigma(x) \approx\left(\vec{a}_{i} \vec{a}_{i}^{\prime}\right) * \sigma(x): \mathrm{s} .
$$

for $1 \leq i \leq n$, where $\vec{a}_{i} \in \mathbb{A}^{(n)}$ is an ordering of $\bar{a}_{i}$ and $\vec{a}_{i}^{\prime} \in \mathbb{A}^{(n)}$ is a tuple of the same size such that $\operatorname{supp}\left(\vec{a}_{i}^{\prime}\right) \#\left(\nabla^{\prime}, \bar{a}_{i}, \sigma(x)\right)$. 


$$
\begin{aligned}
& (\mathrm{REFL}) \frac{\nabla \vdash t \approx t^{\prime}: \mathrm{s}}{\nabla \vdash t \approx t: \mathrm{s}} \nabla \in \mathrm{FE}_{\Sigma}, t \in \Sigma_{\mathrm{s}}\left(\nabla^{:}\right) \quad\left(\mathrm{SYMM}^{o}\right) \frac{\nabla \vdash t^{\prime} \approx t: \mathrm{s}}{\nabla+{ }^{\prime}} \\
& \left(\operatorname{TRANS}^{o}\right) \frac{\nabla \vdash t \approx t^{\prime}: \mathrm{s} \quad \nabla \vdash t^{\prime} \approx t^{\prime \prime}: \mathrm{s}}{\nabla \vdash t \approx t^{\prime \prime}: \mathrm{s}} \quad\left(\mathrm{WEAK}^{o}\right) \frac{\nabla \vdash t \approx t^{\prime}: \mathrm{s}}{\nabla^{\prime} \vdash t \approx t^{\prime}: \mathrm{s}} \nabla \leq \nabla^{\prime} \in \mathrm{FE}_{\Sigma} \\
& \left(\operatorname{SUBST}^{o}\right) \frac{\nabla^{\prime} \vdash \sigma \approx \sigma^{\prime} \quad \nabla^{\prime} \vdash \sigma: \nabla \quad \nabla \vdash t \approx t^{\prime}: \mathrm{s}}{\nabla^{\prime} \vdash t\{\sigma\} \approx t^{\prime}\left\{\sigma^{\prime}\right\}: \mathrm{s}} \sigma, \sigma^{\prime}: \nabla: \rightarrow\left(\nabla^{\prime}\right):
\end{aligned}
$$

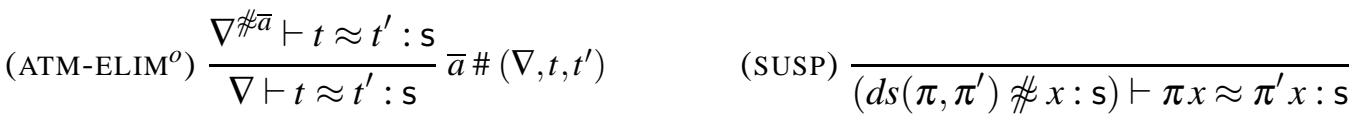

Figure 2: Proof rules for NEoL

Theorem 4.3. If $\mathbb{T}$ is a NEoL-theory (and hence a NEL-theory) then $\nabla \vdash_{\mathbb{T}}^{o} t \approx t^{\prime}: \mathrm{s}$ implies $\nabla \vdash_{\mathbb{T}} t \approx t^{\prime}: \mathrm{s}$.

Proof. We need only check that each of the rules for NEoL of Fig. 2 can be derived from the rules for NEL of Fig. 1. (REFL) and (SUSP) are also rules of Fig. 1, while $\left(\mathrm{SYMM}^{o}\right)$, $\left(\mathrm{TRANS}^{\circ}\right),\left(\mathrm{WEAK}^{o}\right)$ and $\left(\mathrm{ATM}^{\mathrm{ELIM}}{ }^{o}\right.$ ) are clearly special cases of the corresponding rules. $\left(\mathrm{SUBST}^{o}\right)$ is a special case of (SUBST), as (9) is equivalent to the usual condition $\nabla^{\prime} \vdash \bar{a}_{i} \# \sigma\left(x_{i}\right):$ s by Lem. 3.8 .

The next three lemmas relate logical consequence for NEoL (Def. 4.2) with the Perm-actions on terms (2) and (4).

Lemma 4.4. Given a NEoL-theory $\mathbb{T}, \nabla \vdash_{\mathbb{T}}^{o} t \approx t^{\prime}: \mathrm{s}$ implies $\nabla \vdash_{\mathbb{T}}^{o} \pi * t \approx \pi * t^{\prime}: \mathrm{s}$.

Proof.

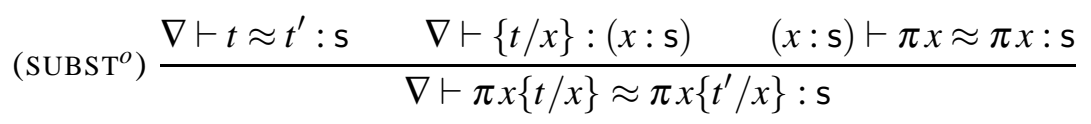

Lemma 4.5. Given a NEoL-theory $\mathbb{T}, \nabla \vdash_{\mathbb{T}}^{o} t \approx t^{\prime}: \mathrm{s}$ implies $\pi \cdot \nabla \vdash_{\mathbb{T}}^{o} \pi \cdot t \approx \pi \cdot t^{\prime}: \mathrm{s}$.

Proof. By Lem. 3.4(ii) this result may be attained via $\left(\mathrm{SuSP}^{o}\right)$ :

$$
\frac{\pi \cdot \nabla \vdash\left(\pi^{-1}-\right): \nabla \quad \nabla \vdash \pi * t \approx \pi * t^{\prime}: \mathrm{s}}{\pi \cdot \nabla \vdash(\pi * t)\left\{\pi^{-1}-\right\} \approx\left(\pi * t^{\prime}\right)\left\{\pi^{-1}-\right\}: \mathrm{s}}
$$

The second premise follows by Lem. 4.4. Now take $\nabla$ as (5) and for $1 \leq i \leq n$ let $\vec{a}_{i}$ be an ordering of $\bar{a}_{i}$ and $\vec{a}_{i}^{\prime}$ be a suitably fresh tuple of the same size. Then $\left(\pi \cdot \bar{a}_{i} \cup \operatorname{supp}\left(\vec{a}_{i}^{\prime}\right) \not \nVdash x_{i}: s_{i}\right) \vdash \pi^{-1} x_{i} \approx\left(\vec{a}_{i} \vec{a}_{i}^{\prime}\right) \pi^{-1} x_{i}: s_{i}$ for each $i$ by (SUSP); applying (WEAK ${ }^{o}$ ) gives us $(\pi \cdot \nabla) \not \nLeftarrow$ supp $\left(\vec{a}_{i}^{\prime}\right) \vdash \pi^{-1} x_{i} \approx\left(\vec{a}_{i} \vec{a}_{i}^{\prime}\right) \pi^{-1} x_{i}: \mathrm{s}_{i}$, which yields the first premise.

Lemma 4.6. Given $\nabla \in \mathrm{FE}_{\Sigma}, t \in \Sigma_{\mathbf{s}}\left(\nabla^{:}\right)$, and $d s\left(\pi, \pi^{\prime}\right) \# t$,

$$
\nabla^{\not \nLeftarrow d s\left(\pi, \pi^{\prime}\right)} \vdash_{\mathbb{T}}^{o} \pi * t \approx \pi^{\prime} * t: \mathrm{s}
$$


Proof. By induction on the structure of $t$.

Suspensions: Say $t=\pi^{\prime \prime} x$. By our freshness assumption $d s\left(\pi, \pi^{\prime}\right)=d s\left(\pi \pi^{\prime \prime}, \pi^{\prime} \pi^{\prime \prime}\right)$, so by (SUSP) $d s\left(\pi, \pi^{\prime}\right) \not \notin x: \mathrm{s} \vdash \pi \pi^{\prime \prime} x \approx \pi^{\prime} \pi^{\prime \prime} x: \mathrm{s}$; the result then follows by $\left(\mathrm{WEAK}^{o}\right)$.

Constructed terms: if $t=o p t_{1} \cdots$ then $\pi * t=\left((\pi \cdot o p) x_{1} \cdots\right)\{\sigma\}$, where $\sigma$ maps each $x_{i} \mapsto \pi * t_{i}$. Similarly $\pi^{\prime} * t=\left(\left(\pi^{\prime} \cdot o p\right) x_{1} \cdots\right)\left\{\sigma^{\prime}\right\}$, where $\sigma^{\prime}$ maps each $x_{i} \mapsto \pi^{\prime} * t_{i} . \pi \cdot o p=\pi^{\prime} \cdot o p$ by our freshness assumption and Lem. 2.3 (ii). To apply $\left(\mathrm{SUBST}^{o}\right)$ to get our result we need only then show $\nabla \not k d s\left(\pi, \pi^{\prime}\right) \vdash$ $\sigma \approx \sigma^{\prime}$; or for each $i, \nabla \sharp d d s\left(\pi, \pi^{\prime}\right) \vdash \pi * t_{i} \approx \pi^{\prime} * t_{i}: \mathrm{s}_{i}$. These judgements follow by induction.

It is a fact about NEL that from $\nabla \vdash_{\mathbb{T}} \bar{a} \not \nVdash t: \mathrm{s}$ we can infer $\nabla \vdash_{\mathbb{T}} \bar{a}^{\prime} \not Æ t: \mathrm{s}$ for $\bar{a}^{\prime} \subseteq \bar{a}$. The next Lemma gives the corresponding result for NEoL.

Lemma 4.7. Suppose we have an NEoL-theory $\mathbb{T}$, freshness environment $\nabla \in \mathrm{FE}_{\Sigma}$, term $t \in \Sigma_{\mathrm{s}}\left(\nabla^{*}\right)$ and lists of atoms $\vec{a}, \vec{b} \in \mathbb{A}^{(n)}$ such that $\operatorname{supp}(\vec{b}) \#(\vec{a}, t)$. Now suppose that $\vec{a}^{\prime}, \vec{b}^{\prime} \in \mathbb{A}^{(m)}$ for some $m \leq n$, with $\operatorname{supp}\left(\vec{a}^{\prime}\right) \subseteq \operatorname{supp}(\vec{a})$ and $\operatorname{supp}\left(\vec{b}^{\prime}\right) \subseteq \operatorname{supp}(\vec{b})$. Then

$$
\nabla \not \text { supp }(\vec{b}) \vdash_{\mathbb{T}}^{o} t \approx(\vec{a} \vec{b}) * t: \mathrm{s} \Rightarrow \nabla \not \text { supp }\left(\vec{b}^{\prime}\right) \vdash_{\mathbb{T}}^{o} t \approx\left(\vec{a}^{\prime} \vec{b}^{\prime}\right) * t: \mathrm{s}
$$

Proof. ( $\left.\operatorname{supp}(\vec{a}) \cup \operatorname{supp}\left(\vec{b}^{\prime}\right) \not Æ x: \mathrm{s}\right) \vdash x \approx\left(\vec{a}^{\prime} \vec{b}^{\prime}\right) x: \mathrm{s}$ by (SUSP). We wish to use ( $\mathrm{SUBST}^{o}$ ) to conclude that $\nabla \not$ supp $\left(\vec{b}^{\prime}\right) \vdash_{\mathbb{T}}^{o} x\{t / x\} \approx\left(\left(\vec{a}^{\prime} \vec{b}^{\prime}\right) x\right)\{t / x\}: \mathrm{s}$; for this substitution to occur we must prove that

$$
\nabla \not \text { supp }\left(\vec{b}^{\prime}\right) \vdash\{t / x\}:\left(\operatorname{supp}(\vec{a}) \cup \operatorname{supp}\left(\vec{b}^{\prime}\right) \not \sharp x: \mathrm{s}\right) .
$$

Now take fresh $\vec{c} \in \mathbb{A}^{(n)}, \vec{c}^{\prime} \in \mathbb{A}^{(m)}$. By Lem. 4.6 we have

$$
\nabla \not k \operatorname{supp}\left(\vec{b}^{\prime}\right) \cup \operatorname{supp}(\vec{c}) \cup \operatorname{supp}\left(\vec{c}^{\prime}\right) \vdash(\vec{a} \vec{c}) * t \approx(\vec{a} \vec{c})\left(\vec{b}^{\prime} \vec{c}^{\prime}\right) * t: \mathrm{s} \text {. }
$$

Applying Lem. 4.5 and $\left(\right.$ WEAK $\left.^{\circ}\right)$ to our hypothesis gives us

$$
\nabla \nVdash \operatorname{supp}\left(\vec{b}^{\prime}\right) \cup \operatorname{supp}(\vec{c}) \cup \operatorname{supp}\left(\vec{c}^{\prime}\right) \vdash t \approx(\vec{a} \vec{c}) * t: \mathrm{s} \text {. }
$$

Combining (11) and (12) with $\left(\operatorname{TRANS}^{\circ}\right)$ gives us

$$
\nabla \not \operatorname{supp}\left(\vec{b}^{\prime}\right) \cup \operatorname{supp}(\vec{c}) \cup \operatorname{supp}\left(\vec{c}^{\prime}\right) \vdash t \approx(\vec{a} \vec{c})\left(\vec{b}^{\prime} \vec{c}^{\prime}\right) * t: \mathrm{s}
$$

which is equivalent to (10) as required.

Definition 4.8. Given a NEL-theory $\mathbb{T}$, let $\mathbb{T}^{o}$ be the NEoL-theory produced by replacing each axiom of the form (6) by the axioms

$$
\nabla \vdash t \approx t^{\prime}: \mathrm{s} \text { and } \nabla \not \nVdash \operatorname{supp}\left(\vec{a}^{\prime}\right) \vdash t \approx\left(\vec{a} \vec{a}^{\prime}\right) * t: \mathrm{s}
$$

where $\vec{a} \in \mathbb{A}^{(n)}$ is an ordering of $\bar{a}$ and $\vec{a}^{\prime} \in \mathbb{A}^{(n)}$ is a tuple of the same size such that $\operatorname{supp}\left(\vec{a}^{\prime}\right) \#(\nabla, \bar{a}, t)$. (6) and (13) are equivalent for NEL by Lem. 3.8.

Theorem 4.9. Let $\mathbb{T}$ be a NEL-theory. Then $\nabla \vdash_{\mathbb{T}} \bar{a} \not t t \approx t^{\prime}: \mathrm{s}$ implies that $\nabla \vdash_{\mathbb{T}^{o}}^{o} t \approx t^{\prime}: \mathrm{s}$ and $\nabla \nVdash \operatorname{supp}\left(\vec{a}^{\prime}\right) \vdash_{\mathbb{T}^{o}}^{o} t \approx\left(\vec{a} \vec{a}^{\prime}\right) * t: \mathrm{s}$, where $\vec{a} \in \mathbb{A}^{(n)}$ is an ordering of $\bar{a}$ and $\vec{a}^{\prime} \in \mathbb{A}^{(n)}$ is a tuple of the same size such that $\operatorname{supp}\left(\vec{a}^{\prime}\right) \#(\nabla, \bar{a}, t)$.

Therefore by Lem. 3.8 and Thm. 4.3 NEL and NEoL are equivalent. 
Proof. Given a $\mathbb{T}$-axiom the corresponding NEoL-judgement is a $\mathbb{T}^{o}$-axiom by Def. 4.8 , The proof then proceeds by induction on the rules of Fig. 1, showing that the corresponding NEoL-judgements may be derived by the rules of Fig. 2

This result is immediate for (REFL) and (SUSP), which are also rules for NEoL. (WEAK) and (ATM-ELIM) follow easily by applications of $\left(\mathrm{WEAK}^{\circ}\right)$ and $\left(\mathrm{ATM}^{\mathrm{ELIM}}{ }^{\circ}\right)$.

(SYMM): Applying the induction hypothesis gives us $\nabla \vdash t \approx t^{\prime}: \mathrm{s}$ and $\nabla \not 4 \operatorname{supp}\left(\vec{a}^{\prime}\right) \vdash t \approx\left(\vec{a} \vec{a}^{\prime}\right) * t: \mathrm{s}$. $\nabla \vdash t^{\prime} \approx t: \mathrm{s}$ by $\left(\mathrm{SYMM}^{o}\right)$. Now $\nabla \vdash\left(\vec{a} \vec{a}^{\prime}\right) * t \approx\left(\vec{a} \vec{a}^{\prime}\right) * t^{\prime}: \mathrm{s}$ by Lem. 4.4, so by $\left(\mathrm{WEAK}^{o}\right)$ and $\left(\mathrm{TRANS}^{o}\right)$ we have $\nabla \not k \operatorname{supp}\left(\vec{a}^{\prime}\right) \vdash t^{\prime} \approx\left(\vec{a} \vec{a}^{\prime}\right) * t^{\prime}: \mathrm{s}$.

(TRANS): The induction hypothesis gives us $\nabla \vdash t \approx t^{\prime}: \mathrm{s}, \nabla \not s u p p\left(\vec{a}_{1}^{\prime}\right) \vdash t \approx\left(\vec{a}_{1} \vec{a}_{1}^{\prime}\right) * t: \mathrm{s}, \nabla \vdash t^{\prime} \approx t^{\prime \prime}: \mathrm{s}$ and $\nabla \not$ supp $\left(\vec{a}_{2}^{\prime}\right) \vdash t^{\prime} \approx\left(\vec{a}_{2} \vec{a}_{2}^{\prime}\right) * t^{\prime}: \mathrm{s}$, where $\vec{a}_{1}, \vec{a}_{2}$ are orderings of $\bar{a}_{1}, \bar{a}_{2}$ respectively, and $\vec{a}_{1}^{\prime}, \vec{a}_{2}^{\prime}$ are fresh tuples of the same sizes. $\nabla \vdash t \approx t^{\prime \prime}: \mathrm{s}$ by $\left(\mathrm{TRANS}^{\circ}\right)$. Now suppose $\bar{a}_{1} \# \bar{a}_{2}$ (if they are not disjoint we use Lem. 4.7 to weaken one side until they are), and use successive applications of (WEAK $\left.{ }^{\circ}\right)$, Lem. 4.4 and $\left(\right.$ TRANS $\left.^{\circ}\right)$ :

$$
\begin{aligned}
\nabla \# \operatorname{supp}\left(\vec{a}_{1}^{\prime}\right) \cup \operatorname{supp}\left(\vec{a}_{2}^{\prime}\right) \vdash_{\mathbb{T}}^{o} t & \approx\left(\vec{a}_{1} \vec{a}_{1}^{\prime}\right) * t \\
& \approx\left(\vec{a}_{1} \vec{a}_{1}^{\prime}\right) * t^{\prime} \\
& \approx\left(\vec{a}_{1} \vec{a}_{1}^{\prime}\right)\left(\vec{a}_{2} \vec{a}_{2}^{\prime}\right) * t^{\prime} \\
& \approx\left(\vec{a}_{1} \vec{a}_{1}^{\prime}\right)\left(\vec{a}_{2} \vec{a}_{2}^{\prime}\right) * t .
\end{aligned}
$$

(SUBST): By the induction hypothesis $\nabla \vdash t \approx t^{\prime}: \mathrm{s}$ and $\nabla \not \sharp \operatorname{supp}\left(\vec{a}^{\prime}\right) \vdash t \approx\left(\vec{a} \vec{a}^{\prime}\right) * t: \mathrm{s}$, and if $\nabla$ is as (5) then for $1 \leq i \leq n$ we have $\nabla^{\prime} \vdash \sigma\left(x_{i}\right) \approx \sigma^{\prime}\left(x_{i}\right): \mathrm{s}_{i}$ and $\left(\nabla^{\prime} \not \sharp\right.$ supp $\left(\vec{a}_{i}^{\prime}\right) \vdash \sigma\left(x_{i}\right) \approx\left(\vec{a}_{i} \vec{a}_{i}^{\prime}\right) * \sigma\left(x_{i}\right): \mathrm{s}_{i}$ where $\vec{a}_{i}$ is an ordering of $\bar{a}_{i}$ and $\vec{a}_{i}^{\prime}$ is a fresh tuple of the same size. $\nabla \vdash t\{\sigma\} \approx t^{\prime}\left\{\sigma^{\prime}\right\}:$ s by $\left(\mathrm{SuBST}^{o}\right)$. Now $\left(\vec{a} \vec{a}^{\prime}\right) *(t\{\sigma\})=\left(\left(\vec{a} \vec{a}^{\prime}\right) * t\right)\{\sigma\}$ by Lem. 3.4 (i), so we look to apply $\left(\mathrm{SUBST}^{o}\right)$ :

$$
\frac{\left(\nabla^{\prime}\right)^{\not \notin \operatorname{supp}\left(\vec{a}^{\prime}\right)} \vdash \sigma: \nabla^{\not \sharp \operatorname{supp}\left(\vec{a}^{\prime}\right)} \quad \nabla^{\not \nVdash \operatorname{supp}\left(\vec{a}^{\prime}\right)} \vdash t \approx\left(\vec{a} \vec{a}^{\prime}\right) * t: \mathrm{s}}{\left(\nabla^{\prime}\right)^{\not \text { supp }\left(\vec{a}^{\prime}\right)} \vdash t\{\sigma\} \approx\left(\left(\vec{a} \vec{a}^{\prime}\right) * t\right)\{\sigma\}: \mathrm{s}}
$$

The second premise is among our hypotheses, while the first follows from ( $\left.\mathrm{SUBST}^{o}\right)$ for each $i$ :

$$
\begin{gathered}
\nabla^{\prime \prime} \vdash \sigma\left(x_{i}\right) \approx\left(\vec{a}_{i} \vec{a}_{i}^{\prime}\right) * \sigma\left(x_{i}\right): \mathrm{s}_{i} \\
\frac{\nabla^{\prime \prime} \vdash\left\{\sigma\left(x_{i}\right) / x\right\}:\left(\operatorname{supp}\left(\vec{a}^{\prime}\right) \cup \operatorname{supp}\left(\vec{a}^{\prime \prime}\right) \not Æ x: \mathrm{s}_{i}\right)}{\left(\operatorname{supp}\left(\vec{a}^{\prime}\right) \cup \operatorname{supp}\left(\vec{a}^{\prime \prime}\right) \not Æ x: \mathrm{s}\right) \vdash x \approx\left(\vec{a}^{\prime} \vec{a}^{\prime \prime}\right) x: \mathrm{s}_{i}} \\
\nabla^{\prime \prime} \vdash x\left\{\sigma\left(x_{i}\right) / x\right\} \approx\left(\vec{a}^{\prime} \vec{a}^{\prime \prime}\right) x\left\{\left(\vec{a}_{i} \vec{a}_{i}^{\prime}\right) * \sigma\left(x_{i}\right) / x\right\}: \mathrm{s}_{i}
\end{gathered}
$$

where $\nabla^{\prime \prime}=\left(\nabla^{\prime}\right) \not$ supp $\left(\vec{a}^{\prime}\right) \cup \operatorname{supp}\left(\vec{a}^{\prime \prime}\right) \cup \operatorname{supp}\left(\vec{a}_{i}^{\prime}\right)$ and $\vec{a}^{\prime \prime}$ is a fresh copy of $\vec{a}^{\prime}$. The first premise here follows from our hypotheses and $\left(\mathrm{WEAK}^{\circ}\right)$; the second follows by Lem. 4.6, and the third by (SUSP).

(ATM-INTRO): By the induction hypothesis $\nabla \vdash t \approx t^{\prime}: \mathrm{s}$ and $\nabla \not$ supp $\left(\vec{b}^{\prime}\right) \vdash t \approx\left(\vec{a}^{\prime} \vec{b}^{\prime}\right) * t: \mathrm{s}$, where $\vec{a}^{\prime}$ is an ordering of $\bar{a}^{\prime}$ and $\vec{b}^{\prime}$ is a fresh tuple of the same size. $\nabla \sharp k \bar{a} \vdash t \approx t^{\prime}$ by $\left(\right.$ WEAK $\left.^{o}\right)$. We need to prove that $\nabla^{\prime} \vdash t \approx(\vec{a} \vec{b})\left(\vec{a}^{\prime} \vec{b}^{\prime}\right) * t: \mathrm{s}$, where $\nabla^{\prime}=\nabla \nVdash \vec{a} \cup \operatorname{supp}(\vec{b}) \cup \operatorname{supp}\left(\vec{b}^{\prime}\right), \vec{a}$ is an ordering of $\bar{a}$ and $\vec{b}$ is a fresh copy. Apply $\left(\operatorname{SuBST}^{o}\right)$ :

$$
\frac{\nabla^{\prime} \vdash t \approx\left(\vec{a} \vec{b}^{\prime}\right) * t: \mathrm{s} \quad \nabla^{\prime} \vdash\{t / x\}:(\bar{a} \cup \operatorname{supp}(\vec{b}) \not Æ x: \mathrm{s}) \quad(\bar{a} \cup \operatorname{supp}(\vec{b}) \not Æ x: \mathrm{s}) \vdash x \approx(\vec{a} \vec{b}) x: \mathrm{s}}{\nabla^{\prime} \vdash x\{t / x\} \approx((\vec{a} \vec{b}) x)\left\{\left(\vec{a} \vec{b}^{\prime}\right) * t / x\right\}: \mathrm{s}}
$$

The first premise follows from our hypothesis and $\left(\mathrm{WEAK}^{\circ}\right)$; the second follows by Lem. 4.6, and the third by (SUSP).

$$
\text { (\#゙-EQUIVAR): }\left(\bar{a} \cup \operatorname{supp}\left(\vec{a}^{\prime}\right) \not Æ x: \mathrm{s}\right) \vdash \pi x \approx\left(\pi \cdot \vec{a} \vec{a}^{\prime}\right) \pi x: \mathrm{s} \text { by (SUSP). }
$$




$$
\frac{d s\left(\pi, \pi^{\prime}\right) \not Æ x: \mathrm{s} \in \nabla}{\nabla \vdash \pi x \approx \pi^{\prime} x: \mathrm{s}} \quad \frac{\nabla \vdash t_{1} \approx t_{1}^{\prime}: \mathrm{s}_{1} \quad \cdots \quad \nabla \vdash t_{n} \approx t_{n}^{\prime}: \mathrm{s}_{n}}{\nabla \vdash o p t_{1} \cdots t_{n} \approx o p t_{1}^{\prime} \cdots t_{n}^{\prime}: \mathrm{s}}
$$

Figure 3: Syntax-directed rules for Nominal Equality

\section{The Empty Theory}

In standard equational logic, provable equality corresponds to literal syntactic equality in the empty theory $\emptyset$ without axioms. This is not the case for NEoL because of the presence of freshness environments and suspensions. In this section we give a simple syntax-directed description of equality in the empty NEoL-theory. It is then straightforward to extend this to a description of freshness in the empty NELtheory.

Definition 5.1. Fig. 3 provides syntax-directed rules for Nominal Equality. The notation ' $d s\left(\pi, \pi^{\prime}\right) \not \notin x$ : $\mathrm{s} \in \nabla^{\prime}$ means that $\nabla(x)=(\bar{a}, \mathrm{~s})$ for $\bar{a} \supseteq d s\left(\pi, \pi^{\prime}\right)$.

Theorem 5.2. The rules for NEoL of Fig. 2 imply the syntax-directed rules of Fig. 3

Proof. The suspension case hold by (SUSP) and $\left(\mathrm{WEAK}^{o}\right)$; the constructed term case by $\left(\mathrm{SUBST}^{o}\right)$.

Lemma 5.3. Say we have permutations $\pi, \pi^{\prime} \in$ Perm and a finite list $\vec{a}$ of atoms so that $d s\left(\pi, \pi^{\prime}\right) \subseteq$ $\operatorname{supp}(\vec{a})$. Let $\vec{a}^{\prime}$ be a list of fresh atoms of the same size. Then if we can derive

$$
\nabla \vdash t \approx t^{\prime}: \mathrm{s} \text { and } \nabla \text { supp }\left(\vec{a}^{\prime}\right) \vdash t \approx\left(\vec{a} \vec{a}^{\prime}\right) * t: \mathrm{s}
$$

by the syntax-directed rules of Fig. 3 then we can also derive

$$
\nabla \vdash \pi * t \approx \pi^{\prime} * t^{\prime}: \mathrm{s}
$$

Proof. Suspensions: Say $t=\xi x$ and $t^{\prime}=\xi^{\prime} x$, so $d s\left(\xi, \xi^{\prime}\right) \not 火 x: \mathrm{s} \in \nabla$ and $d s\left(\xi,\left(\vec{a} \vec{a}^{\prime}\right) \xi\right) \not t x: \mathrm{s} \in$ $\nabla \not$ supp $\left(\vec{a}^{\prime}\right)$. We must prove that $\nabla \vdash \pi \xi x \approx \pi^{\prime} \xi^{\prime} x$, i.e. that $d s\left(\pi \xi, \pi^{\prime} \xi^{\prime}\right) \not \nVdash x: \mathrm{s} \in \nabla$. Take $a \in d s\left(\pi \xi, \pi^{\prime} \xi^{\prime}\right)$. If $a \in d s\left(\xi, \xi^{\prime}\right)$ we're done by our first assumption. But if $\xi(a) \in d s\left(\pi, \pi^{\prime}\right)$ then $\xi(a) \in \operatorname{supp}(\vec{a})$, so $a \in d s\left(\xi,\left(\vec{a} \vec{a}^{\prime}\right) \xi\right)$ and by our second assumption $a \not t x: \mathrm{s} \in \nabla \not \sharp \operatorname{supp}\left(\vec{a}^{\prime}\right)$. But $\vec{a}^{\prime}$ was chosen fresh, so $a \not t x \in \nabla$.

Constructed terms: Let $t=o p t_{1} \cdots$ and $t^{\prime}=o p t_{1}^{\prime} \cdots$, so for all $i, \nabla \vdash t_{i} \approx t_{i}^{\prime}: \mathrm{s}_{i}, \nabla \sharp$ supp $\left(\vec{a}^{\prime}\right) \vdash t_{i} \approx$ $\left(\vec{a} \vec{a}^{\prime}\right) * t_{i}: s_{i}$ and $o p=\left(\vec{a} \vec{a}^{\prime}\right) \cdot o p$. We must prove that $\nabla \vdash(\pi \cdot o p)\left(\pi * t_{1}\right) \cdots \approx\left(\pi^{\prime} \cdot o p\right)\left(\pi^{\prime} * t_{1}^{\prime}\right) \cdots$. Now $\operatorname{supp}\left(\vec{a}^{\prime}\right) \#$ op, so by Lem. 2.3 (i) $\operatorname{supp}(\vec{a}) \#\left(\vec{a} \vec{a}^{\prime}\right) \cdot o p=o p$. $d s\left(\pi, \pi^{\prime}\right) \subseteq \operatorname{supp}(\vec{a})$, so by Lem. 2.3(ii) $\pi \cdot o p=\pi^{\prime} \cdot o p$. Finally, $\nabla \vdash \pi * t_{i} \approx \pi^{\prime} * t_{i}^{\prime}: \mathrm{s}_{i}$ follows by induction.

Theorem 5.4. Suppose $\nabla \vdash_{\emptyset}^{o} t \approx t^{\prime}$ : s by the rules for NEoL of Fig. 2 Then $\nabla \vdash t \approx t^{\prime}: \mathrm{s}$ by the syntax-directed rules of Fig. 3 .

Therefore by Thm. 5.2 the syntax-directed rules coincide with the empty NEoL-theory.

Proof. By induction on the rules of Fig. 2 ,

(SUSP) follows immediately from the suspension case of Fig. 3. (REFL) follows by an easy induction on the structure of $t .\left(\mathrm{SYMM}^{o}\right),\left(\mathrm{WEAK}^{o}\right)$ and $\left(\mathrm{ATM}^{\mathrm{ETLIM}}{ }^{o}\right)$ are also straightforward.

$\left(\right.$ TRANS $\left.^{o}\right)$ : Say $t=\pi x, t^{\prime}=\pi^{\prime} x$ and $t^{\prime \prime}=\pi^{\prime \prime} x$, so $d s\left(\pi, \pi^{\prime}\right) \not t x: \mathrm{s}, d s\left(\pi^{\prime}, \pi^{\prime \prime}\right) \not t x: \mathrm{s} \in \nabla$. We need to show that $d s\left(\pi, \pi^{\prime \prime}\right) \not \notin x: \mathrm{s} \in \nabla$, so take $a \in d s\left(\pi, \pi^{\prime \prime}\right)$. If $a \in d s\left(\pi, \pi^{\prime}\right)$ we are done by our first assumption, 
but if $\pi(a)=\pi^{\prime}(a)$ then $a \in d s\left(\pi^{\prime}, \pi^{\prime \prime}\right)$ so we are done by our second assumption. The constructed term case is an easy induction.

$\left(\operatorname{suBST}^{o}\right)$ : Say $\nabla \vdash t \approx t^{\prime}$ is $\nabla \vdash \pi x \approx \pi x: \mathrm{s}, \sigma(x)=\xi y$ and $\sigma^{\prime}(x)=\xi^{\prime} y$, so $d s\left(\pi, \pi^{\prime}\right) \not t x: \mathrm{s} \in \nabla$ and $d s\left(\xi, \xi^{\prime}\right) \not t y: s \in \nabla^{\prime}$. The other premise (9) says that $d s\left(\xi,\left(\vec{a} \vec{a}^{\prime}\right) \xi\right) \not \sharp y: s \in\left(\nabla^{\prime}\right) \not$ supp $\left(\vec{a}^{\prime}\right)$ where $\nabla(x)=(\bar{a}, \mathrm{~s}), \vec{a}$ is an ordering of $\bar{a}$, and $\vec{a}^{\prime}$ is a fresh tuple of the same size. We need to prove that $d s\left(\pi \xi, \pi^{\prime} \xi^{\prime}\right) \not \notin y: \mathrm{s} \in \nabla^{\prime}$. Take $a \in d s\left(\pi \xi, \pi^{\prime} \xi^{\prime}\right)$. If $a \in d s\left(\xi, \xi^{\prime}\right)$ we are done, so say $\xi(a) \in d s\left(\pi, \pi^{\prime}\right) \subseteq \bar{a}$. Then $a \in d s\left(\xi,\left(\vec{a} \vec{a}^{\prime}\right) \xi\right)$, so $a \not k y: \mathrm{s} \in\left(\nabla^{\prime}\right) \not k$ supp $\left(\vec{a}^{\prime}\right)$, but $\vec{a}^{\prime}$ was chosen fresh, so we are done.

Now take $t, t^{\prime}$ as above, so $d s\left(\pi, \pi^{\prime}\right) \not t x: \mathrm{s} \in \nabla$ still, but $\sigma(x)=o p t_{1} \cdots$ and $\sigma^{\prime}(x)=o p t_{1}^{\prime} \cdots$. Then for $1 \leq i \leq n, \nabla^{\prime} \vdash t_{i} \approx t_{i}^{\prime}: \mathrm{s}_{i},\left(\nabla^{\prime}\right) \not \sharp s u p p\left(\vec{a}^{\prime}\right) \vdash t_{i} \approx\left(\vec{a} \vec{a}^{\prime}\right) * t_{i}: \mathrm{s}$ and $o p=\left(\vec{a} \vec{a}^{\prime}\right) \cdot o p$, where $\bar{a}, \vec{a}, \vec{a}^{\prime}$ are also as above. $\operatorname{supp}\left(\vec{a}^{\prime}\right) \#$ op implies $\bar{a} \#\left(\vec{a} \vec{a}^{\prime}\right) \cdot$ op $=$ op, so $\pi \cdot$ op $\approx \pi^{\prime} \cdot$ op. $\nabla^{\prime} \vdash \pi * t_{i} \approx \pi * t_{i}^{\prime}$ by Lem. 5.3 .

Finally, if $t, t^{\prime}$ are constructed terms then the induction for $\left(\mathrm{SUBST}^{o}\right)$ is straightforward.

Corollary 5.5. The empty NEL-theory, following the rules of Fig. 1$]$ coincides with the syntax-directed rules of Fig. 3 along with these new rules for freshness:

$$
\frac{\pi^{-1} \cdot \bar{a} \not Æ x: \mathrm{s} \in \nabla}{\nabla \vdash \bar{a} \not Æ \pi x: \mathrm{s}} \quad \frac{\nabla \vdash \bar{a} \not Æ t_{1}: \mathrm{s}_{1} \quad \cdots \quad \nabla \vdash \bar{a} \not Æ t_{n}: \mathrm{s}_{n} \quad \bar{a} \# \text { op }}{\nabla \vdash \bar{a} \not Æ o p t_{1} \cdots t_{n}: \mathrm{s}}
$$

Proof. Lem. 3.8, Thm. 4.9 and Thm. 5.4.

\section{Related Work}

Equational logic for nominal unification. The first notion of equational logic over nominal sets to be developed were the syntax-directed rules of [13, Fig. 2], which were used in the definition of nominal unification. The syntax that directs this definition is based on nominal signatures, which compared to the signatures of Def. 3.1 have a richer sort system and a set, rather than nominal set, of operation symbols.

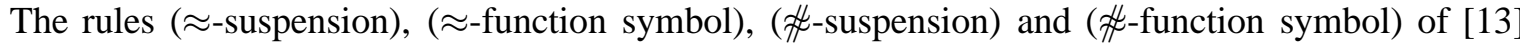
clearly match the syntax-directed rules of Fig. 3 and Cor. 5.5, apart from the premise $\bar{a} \#$ op, which is non-trivial only when $o p$ may have non-empty support. If we add operation symbols for unit, pairing, atoms and atom-abstraction then, via Fig. 3 and Cor. 5.5, we recover all of the rules of [13] except for $(\approx$-abstraction-2) and ( $\not$-abstraction- 1$)$ :

$$
\frac{a \neq a^{\prime} \quad \nabla \vdash t \approx\left(a a^{\prime}\right) * t^{\prime} \quad \nabla \vdash a \not Æ t^{\prime}}{\nabla \vdash a . t \approx a^{\prime} \cdot t^{\prime}} \quad \overline{\nabla \vdash a \not Æ a . t}
$$

where $a . t$ is the atom-abstraction binding $a$ in $t$. These are the rules for $\alpha$-equivalence. Following [3], we may capture these rules by moving from the empty theory to the theory with one axiom

$$
(b \not t x) \vdash a \cdot x \approx b .(a b) x
$$

or equivalently, $(x) \vdash a \not \sharp a . x$.

Nominal Algebra (NA). NA [8] is a logic independently developed to reason about the same properties as NEL, but with some interestingly different design choices. NA is built on nominal signatures, so in the empty theory equality should be, as above, $\alpha$-equivalence over these signatures rather than the weaker equivalence of Sec. 5. NA employs a set, rather than nominal set, of operation symbols, which may make it less expressive than NEL. For example, with NEL one could define a nominal set of operation symbols isomorphic to the nominal set $\mathbb{A}^{(2)}$ of disjoint pairs of atoms; this does not seem to be possible with NA. 
Finally, NA employs a syntax-directed notion of freshness that is weaker than that used by NEL; in particular the transitive property

$$
\frac{\nabla \vdash a \not Æ t: \mathrm{s} \quad \nabla \vdash t \approx t^{\prime}: \mathrm{s}}{\nabla \vdash a \not \sharp t^{\prime}: \mathrm{s}}
$$

does not hold. In [8, Sec. 5] design alternatives for NA were discussed where atom-abstraction sorts were eliminated and the freshness relation strengthened to match that of NEL. However no notion of NA with equality only has been proposed along the lines of NEoL, although it seems likely that such a logic could be defined by working in close analogy with the results of this paper.

Synthetic Nominal Equational Logic (SNEL). Term Equational Systems [6] are a category theoretic account of equational logic, including proof theory. This framework allows equational logic to be naturally generalised from the category of sets to other categories, with proof rules automatically generated in each new setting so long as the new categories obey certain certain constraints. Following NEL and NA, Term Equational Systems were developed in the category of nominal sets, and the resulting logic is called SNEL [6, Sec. 5]. SNEL is another notion of nominal sets with equations only, but no proof was offered that the addition of freshness judgements would not strengthen the logic. The authors were, however, aware of the results presented in this paper, which could be seen as a sanity check on the development of the equation-only SNEL.

It should also be noted that the syntax of SNEL is not entirely in keeping with that which is commonly used in nominal logic, as we have no freshness environments or suspensions. For example, the axiom (8) for $\alpha$-equivalence in the untyped $\lambda$-calculus would be written

$$
[a, b]\{x: 1\} \vdash \operatorname{lam}_{a} x(a) \approx \operatorname{lam}_{b} x(b) .
$$

Here the metavariables explicitly refer to names they may depend on. This differs from the standard mathematical treatment of bound names, which most applications of nominal techniques try to capture. It is an interesting question whether a more standard presentation of equational logic over nominal sets, such as NEL or NA, could be derived in this category theoretic context.

\section{References}

[1] S. N. Burris (1998): Logic for Mathematics and Computer Science. Prentice Hall.

[2] R. Clouston (2009): Equational Logic for Names and Binders. Ph.D. thesis, University of Cambridge. http://cecs.anu.edu.au/ rclouston/Clouston_Thesis.pdf.

[3] R. Clouston (2010): Binding in Nominal Equational Logic. In: MFPS, ENTCS 265, pp. 259-276, doi:10. 1016/j.entcs.2010.08.016.

[4] R. Clouston (2011): Nominal Lawvere Theories. In: WoLLIC, LNCS 6642, pp. 67-83, doi:10.1007/ 978-3-642-20920-8_11.

[5] R. Clouston \& A. M. Pitts (2007): Nominal Equational Logic. ENTCS 172, pp. 223-257, doi:10.1016/j . entcs.2010.08.016.

[6] M. Fiore \& C-K. Hur (2008): Term Equational Systems and Logics. In: MFPS, ENTCS 218, pp. 171-192, doi:10.1016/j.entcs.2008.10.011.

[7] M. J. Gabbay \& A. Mathijssen (2006): One-and-a-halfth-orderLogic. In: PPDP, ACM, pp. 189-200, doi:10. 1145/1140335.1140359.

[8] M. J. Gabbay \& A. Mathijssen (2009): Nominal (Universal) Algebra: Equational Logic with Names and Binding. J. Logic Comput. 19(6), pp. 1455-1508, doi:10.1007/s001650200016. 
[9] M. J. Gabbay \& A. M. Pitts (2002): A New Approach to Abstract Syntax with Variable Binding. FAC 13, pp. 341-363, doi:10.1016/j . entcs .2008.10.011.

[10] F. W. Lawvere (1963): Functorial Semantics of Algebraic Theories. Ph.D. thesis, Columbia University.

[11] A. M. Pitts (2001): Nominal Logic: a First Order Theory of Names and Binding. LNCS 2215, pp. 219-242, doi:10.1007/3-540-45500-0_11.

[12] A. M. Pitts (2006): Alpha-structural Recursion and Induction. J. ACM 53, pp. 459-506, doi:10.1145/ 1147954.1147961.

[13] C. Urban, A. M. Pitts \& M. J. Gabbay (2004): Nominal Unification. TCS 323(1-3), pp. 473-497, doi:10. 1007/s10817-009-9164-3. 